\title{
CARACTERIZAÇÃO SENSORIAL DE LINGUIÇA TOSCANA COM BAIXO TEOR DE SÓDIO E ANÁLISE DO CONSUMO DE CARNE SUÍNA E DERIVADOS NA REGIÃO OESTE DO PARANÁ
}

\author{
DANIELA MIOTTO BERNARDI* \\ JANESCA ALBAN ROMAN**
}

\begin{abstract}
O propósito desse estudo foi avaliar a aceitabilidade, perante o mercado consumidor, de linguiça toscana com baixo teor de sódio, bem como investigar o consumo de carne suína e seus derivados na região oeste do Paraná. Três amostras de linguiça toscana com diferentes concentrações de sódio foram avaliadas por 70 julgadores não treinados, utilizando-se diferentes testes sensoriais: escala hedônica, aceitabilidade e preferência, escala ideal de sabor e intenção de compra. Investigou-se o consumo de carne suína e seus derivados por meio de questionário, contendo 13 questões semi-estruturadas. Todas as amostras apresentaram aceitabilidade acima de $70 \%$ para os atributos avaliados. A amostra preferida, com adição de $50 \%$ de cloreto de potássio, alcançou $79 \%$ de intenção de compra. Também foi verificado que a região oeste do Paraná é potencial consumidora de carne suína, sendo os embutidos mais consumidos do que a carne "in natura".
\end{abstract}

* Nutricionista, Mestre em Alimentos e Nutrição pela Universidade Estadual de Campinas (UNICAMP), Campinas, SP (e-mail: dani_miotto@yahoo.com.br).

** Tecnóloga de Alimentos, Doutora em Alimentos e Nutrição, Docente, Universidade Tecnológica Federal do Paraná (UTFPR), campus Toledo, PR (e-email: roman.janesca@gmail.com). 


\section{INTRODUÇÃo}

A carne constitui importante fonte proteica na dieta, sendo a carne suína a mais consumida em todo o mundo (BRAGAGNOLO e RODRIGUEZ-AMAYA, 2002). O Brasil destaca-se como grande produtor mundial de suínos, entretanto seu consumo per capita é em média 4 vezes menor que o da União Européia (ABIPECS, 2007; FARIA, FERRERA e GARCIA, 2005).

Segundo Ramundo, Couto e Lanzillotti (2005) existe ampla variedade de derivados de carne suína no mercado nacional e na última década, os embutidos (em especial as linguiças frescais) evidenciaram significativa expansão.

A linguiça toscana é o produto cárneo cru, obtido exclusivamente a partir de carne suína, adicionada de gordura suína e de outros ingredientes que atuam na modificação de propriedades como textura, conservação e sabor. Entretanto, tais ingredientes apresentam elevado teor de sódio em sua composição (FERRARI e SOARES, 2003; BRASIL, 2000; ALMEIDA, 2005; RAMUNDO, COUTO E LANZILLOTTI, 2005).

Excesso de sódio, de calorias e de álcool, assim como a falta de cálcio e potássio são os principais fatores nutricionais associados à hipertensão arterial sistêmica (HAS), além da interação de fatores genéticos e ambientais (CABRAL et al., 2003; MOLINA et al., 2003). A HAS apresenta alta prevalência na população brasileira, cerca de 10 a 45\% da população adulta (REIS e COPLE, 1999; CASTRO et al., 1999; MOLINA et al., 2003) e de 1 a 2\% de crianças e adolescentes (KATER e COSTA-SANTOS, 2001). Nesse contexto, destaca-se a necessidade de maior preocupação por parte da indústria alimentícia com os consumidores hipertensos.

$\mathrm{O}$ presente trabalho teve como objetivo avaliar a aceitabilidade, perante o mercado consumidor, de três diferentes amostras de linguiça toscana com baixo teor de sódio, bem como investigar o consumo de carne suína e derivados na região oeste do Paraná.

\section{MATERIAL E MÉTODOS}

\subsection{CRITÉRIOS ÉTICOS E POPULAÇÃO}

A pesquisa foi realizada de acordo com os procedimentos de coleta de dados para trabalhos envolvendo seres humanos. O projeto foi encaminhado ao Comitê de Ética e Pesquisa com Seres Humanos da Faculdade Assis Gurgacz (FAG), recebendo parecer favorável (n99/2007).

A amostra foi constituída por 70 indivíduos com idade acima de 18 anos de ambos os gêneros.

\subsection{QUESTIONÁRIO}

Elaborou-se questionário contendo dados de identificação (idade, gênero, descendência e profissão) e questões envolvendo o consumo e conhecimentos em relação à carne suína e derivados. Também foi abordado o tema hipertensão (Anexo 1).

\subsection{AMOSTRAS}

Três amostras de linguiça toscana com teor reduzido de sódio (LO, L50S, L50SK) foram elaboradas (BERNARDI e ROMAN, 2011):

- $\quad$ LO = linguiça toscana sem adição de sal na formulação, porém com $50 \%$ de aumento na quantidade de pimenta calabresa em relação à formulação padrão (comercial). A amostra L0 continha quantidade de sódio de $102 \mathrm{mg} \mathrm{em} 60 \mathrm{~g}$ de produto, proveniente dos condimentos adicionados como conservantes do produto, bem como da quantidade de sódio naturalmente presente na carne;

- L50S = linguiça toscana com adição de apenas 50\% de sal (cloreto de sódio) em relação à formulação padrão e quantidade padrão de pimenta. A amostra L50S continha $132 \mathrm{mg}$ de sódio em $60 \mathrm{~g}$ de produto;

- $\quad$ L50SK = linguiça toscana com adição de $50 \%$ de sal light (cloreto de potássio) e quantidade de pimenta calabresa aumentada em $50 \%$ em relação à formulação padrão. A amostra L50SK continha $108 \mathrm{mg}$ de sódio em $60 \mathrm{~g}$ de produto; 


\subsection{ANÁLISE SENSORIAL}

As três amostras de linguiça toscana (L0, L50S, L50SK) foram servidas de forma aleatória aos julgadores, em pratos codificados com algarismos de 3 digítos e quantidades padronizadas de aproximadamente $15 \mathrm{~g}$ cada, não havendo repetição das amostras. Um copo com água para a limpeza do palato entre as avaliações foi fornecido aos participantes (MONTEIRO, 1984).

A aceitabilidade das amostras foi avaliada em cabines específicas, utilizando-se diferentes testes sensoriais: escala hedônica, aceitabilidade e preferência, escala ideal de sabor e intenção de compra.

Os atributos cor, aroma, tempero, sabor e consistência foram avaliados por meio da escala hedônica estruturada de 9 pontos, cujos extremos correspondiam a desgostei muitíssimo (1) e gostei muitíssimo (9). O índice de aceitabilidade foi calculado com base na média das notas atribuídas nessa escala (SANTANA et al., 2006):

$$
\begin{aligned}
\mathrm{I}=\frac{\mathrm{C} \times 100}{\mathrm{~B}} \rightarrow \mathrm{C}=\text { nota média obtida para o produto } \\
\rightarrow \mathrm{B}=\text { nota máxima dada ao produto }
\end{aligned}
$$

O teste da escala ideal de sabor foi realizado para verificar a opinião dos julgadores em relação à quantidade de sal presente nas amostras de linguiça toscana. Utilizou-se escala de categoria bipolar de 9 pontos, cujos extremos correspondiam à extremamente menos salgado que o ideal (1) e extremamente mais salgado que o ideal (9) (MONTEIRO, 1984).

Para avaliar a preferência das amostras foi solicitado aos julgadores que anotassem o número da amostra preferida. Em seguida, o participante foi convidado a avaliar sua atitude enquanto consumidor em relação à intenção de compra do produto, utilizado-se escala de categoria bipolar de 5 pontos cujos extremos correspondiam à certamente compraria (1) e certamente não compraria (5) (MONTEIRO, 1984).

\subsection{ANÁLISE ESTATÍSTICA}

Os resultados do questionário e da análise sensorial foram avaliados mediante análise de variância (ANOVA) e as diferenças entre as médias $(p>0,05)$ pelo teste de Tukey, utilizando-se o programa Statística: basic statistica and tables (STATSOFT, 1995)

\section{RESULTADOS E DISCUSSÃO}

\subsection{QUESTIONÁRIO}

A aplicação do questionário envolveu grupo de 70 participantes, entretanto 9 questionários foram excluídos por estarem incompletos. Dos 61 questionários restantes, $37,7 \%$ foram preenchidos por indivíduos do gênero feminino e $62,3 \%$ masculino. A idade dos participantes foi de $39 \pm 12,83$ anos. A descendência dos entrevistados predominante foi italiana $(42,6 \%)$, seguida pela portuguesa $(18,0 \%)$ e alemã $(16,4 \%)$. Com relação à ocupação prevaleceram os profissionais liberais, empregados do comércio e estudantes.

Embora a carne suína seja a mais consumida em todo o mundo, os brasileiros dão preferência para aves e bovinos. A média de consumo de carne suína in natura no Brasil é de $12,1 \mathrm{~kg} / \mathrm{hab} / \mathrm{ano}$, enquanto a média mundial alcança 14,5 kg/hab/ano e na Europa é superior a $40 \mathrm{~kg} / \mathrm{hab} / \mathrm{ano}$ (ABIPECS, 2007). Preconceitos e falta de informação podem ser as causas para o baixo consumo dessa carne no Brasil. Entretanto, o presente estudo mostrou que na região oeste do Paraná existe maior aceitação para esse tipo de carne, uma vez que $98,4 \%$ dos entrevistados afirmaram que consomem carne suína. A maioria deles informou consumo igual ou superior a pelo menos 1 vez por semana, tanto para a carne in natura, como para embutidos em geral (Figura 1).

Ao comparar os resultados obtidos com os encontrados por Faria, Ferreira e Garcia (2006) observou-se frequência de consumo de carne suína "in natura" bem maior na região do Oeste do Paraná do que em Belo Horizonte. Tal fato pode ser explicado pela colonização regional, visto que no oeste paranaense predominam descendentes de italianos e alemães. 


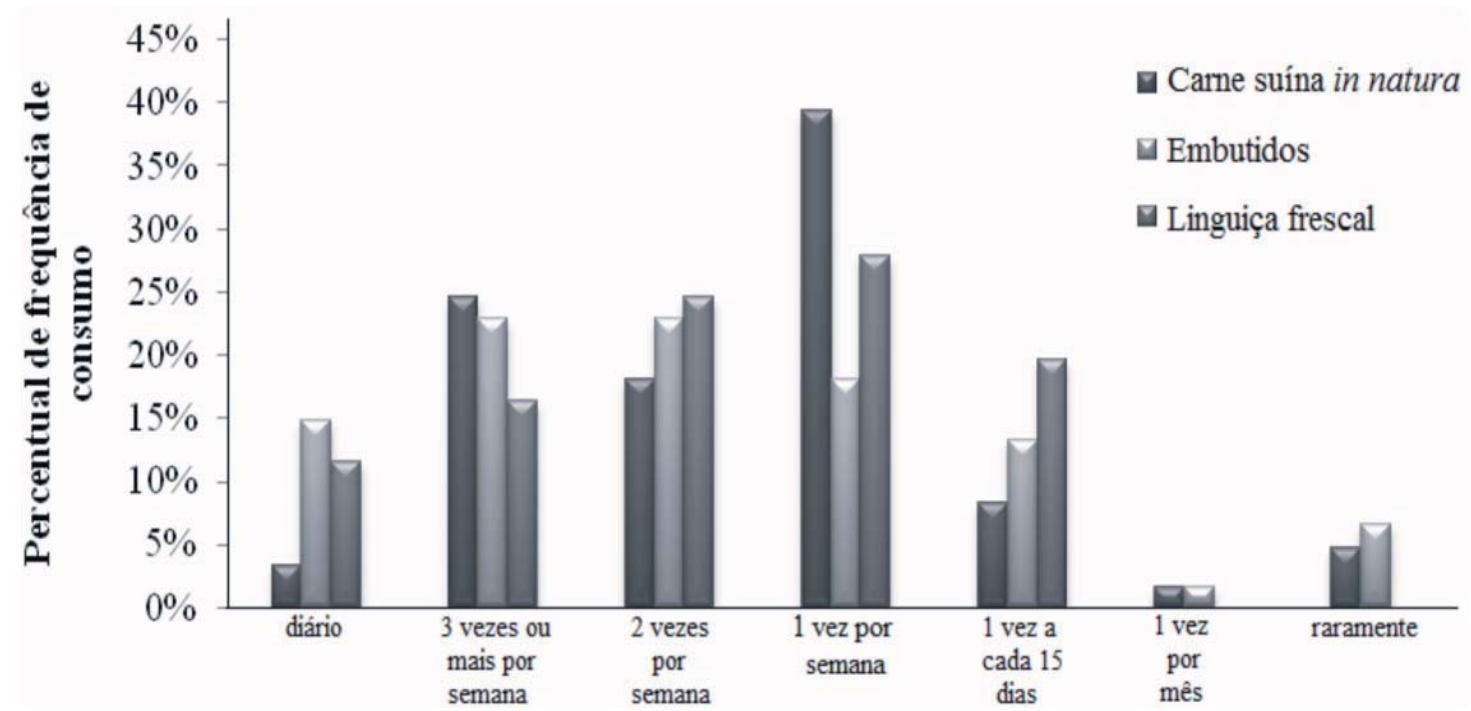

\section{FIGURA 1 - FREQUÊNCIA DE CONSUMO DE CARNE SUÍNA E DERIVADOS}

Entre os produtos derivados de suíno, o salame é o mais consumido, seguido por presunto, costela, lombo, bacon, pernil, linguiça fresca, defumados em geral e outros. Foi observado que o consumo diário de embutidos é maior que da carne in natura, provavelmente devido à maior praticidade e diversificação no preparo de embutidos.

O principal motivo que leva os consumidores à comprarem produtos cárneos obtidos de suínos (Figura 2) foi o "gostar" (40\%), seguido pelo "hábito familiar" (17\%). Confirmando pesquisa realizada por Faria, Ferreira e Garcia (2006), o "preço" (1\%) foi um dos motivos pouco mencionados, bem como "variar o cardápio" (12\%) e "praticidade" (3\%).

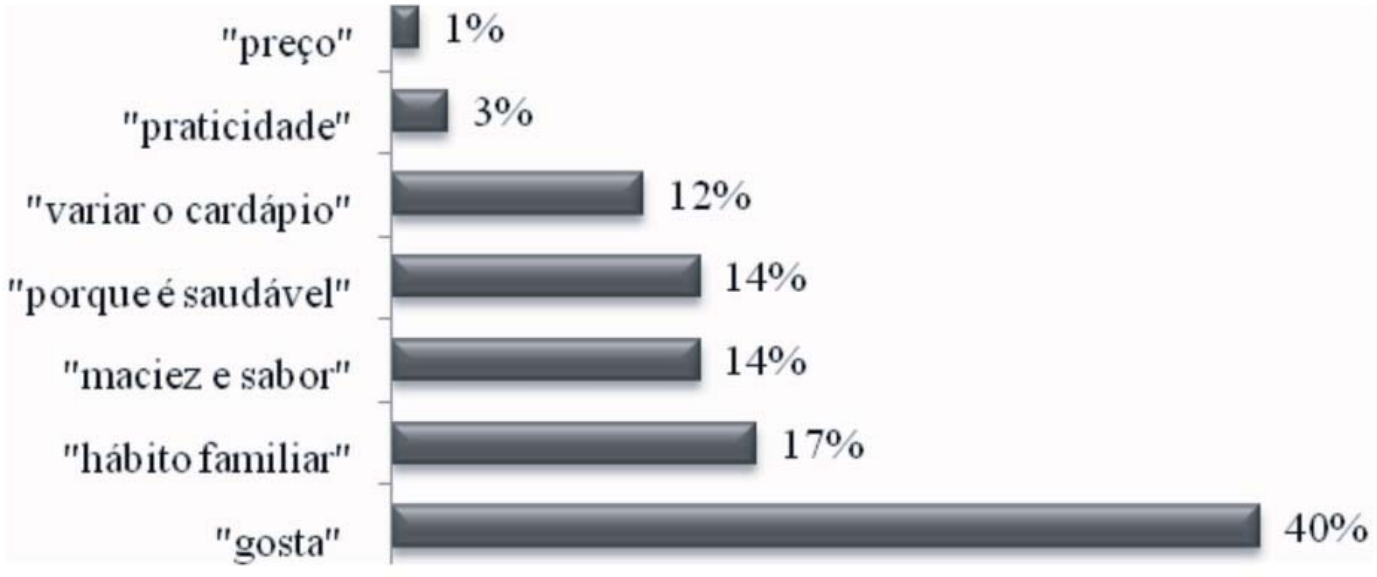


Para 32,8\% dos participantes, a carne suína constitui alimento gorduroso e 37,7\% já ouviram algum tipo de contra indicação para sua ingestão. Apesar de constantes avanços tecnológicos, o consumidor brasileiro ainda demonstra preconceito com relação à carne suína. Os conceitos mais errôneos sobre esse tipo de carne incluem: altos teores de gordura; elevada quantidade de colesterol; e alimento potencialmente perigoso à saúde sob o ponto de vista sanitário (ROPPA, 2002).

Verificou-se alta incidência de hipertensos $(11,48 \%)$ na população avaliada, sendo que $62,3 \%$ dos participante relataram casos de hipertensão na família.

\subsection{ANÁLISE SENSORIAL}

Os julgadores avaliaram as amostras de linguiça toscana com redução dos teores de sódio mediante escala ideal de sabor, preferência, aceitabilidade e intenção de compra.

Com a escala ideal de intensidade de sabor avaliou-se o quanto a alteração de sal nas formulações pode ser perceptível pelos julgadores em relação ao sabor ideal da linguiça toscana (Figura 3).

As duas amostras de linguiça toscana com redução de 50\% de sal, L50S e L50SK, diferiram quanto à quantidade de pimenta calabresa e o tipo de sal adicionados, respectivamente sódio e potássio. No entanto, apresentaram pequenas diferenças quanto ao sabor ideal. O sabor ideal, segundo $54 \%$ dos julgadores corresponde à amostra contendo sal de potássio (L50SK), podendo estar relacionado a maior adição de pimenta calabresa, quando comparada à L50S. A amostra L0 foi a que teve maior redução de sal, sendo que $29 \%$ dos julgadores consideraram-na "ligeiramente menos salgada que o ideal".

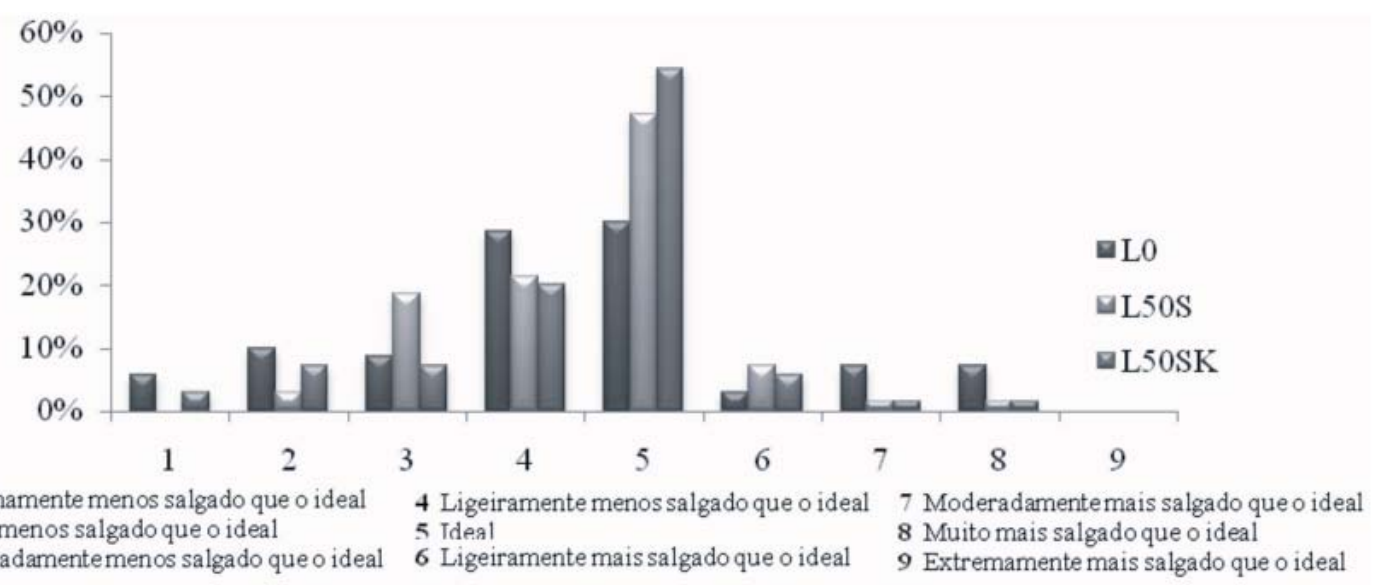

\section{FIGURA 3 - PERCENTUAL OBTIDO NA ESCALA IDEAL DE SABOR PARA AS 3 AMOSTRAS DE LINGUIÇA TOSCANA ELABORADAS COM REDUÇÃO DE SAL, SENDO LO (SEM ADIÇÃO DE SAL), L50S (COM 50\% DE ADIÇÃO DE SAL DE SÓDIO) E L50SK (COM 50\% DE ADIÇÃO DE SAL DE POTÁSSIO)}

Os resultados do teste de preferência e aceitabilidade dos atributos cor, aroma, tempero, sabor e consistência das linguiças estão apresentados na Tabela 1. As médias das notas atribuídas pelos julgadores às amostras degustadas foram semelhantes e estiveram entre 6,3 e 7,9, correspondentes à "gostei ligeiramente e gostei muito" de acordo com a escala hedônica.

Quando comparados os atributos da mesma amostra (linha) observou-se diferença significativa apenas para o produto sem adição de sal (LO). Diferenças estatísticas foram observadas entre o sabor e o tempero em comparação aos demais atributos estudados.

Ao analisar os resultados para a cor, o aroma, o tempero, o sabor e a consistência entre as amostras (coluna), apenas o aroma não diferiu estatisticamente. As linguiças adicionadas de $50 \%$ de sal de sódio (L50S) ou de potássio (L50K) não apresentaram diferenças estatísticas para todos os atributos avaliados, indicando que os julgadores não perceberam a alteração no tipo de 
sal. Apesar das respostas observadas na escala do ideal, a equipe de julgadores considerou ideal o sabor da linguiça contendo sal de potássio.

TABELA 1 - ACEITABILIDADE (\%) DAS AMOSTRAS DE LINGUIÇA COM TEOR REDUZIDO DE SÓDIO, SENDO LO (SEM ADIÇÃO DE SAL), L50S (COM 50\% DE ADIÇÃO DE SAL DE SÓDIO) E L50SK (COM 50\% DE ADIÇÃO DE SAL DE POTÁSSIO)

\begin{tabular}{cccccccc}
\hline \multirow{2}{*}{ Amostras } & Método & \multicolumn{5}{c}{ Atributos } & Média \\
\cline { 3 - 7 } & sensorial & Cor & Aroma & Tempero & Sabor & Consistência \\
\hline \multirow{2}{*}{ LO } & Média das notas & $6,8^{\mathrm{Ba}}$ & $7,2^{\mathrm{Aa}}$ & $6,3^{\mathrm{Bb}}$ & $6,3^{\mathrm{Bb}}$ & $7,0^{\mathrm{Ba}}$ & $\mathbf{6 , 7}$ \\
& IA\% & 76 & 80 & 70 & 70 & 78 & $\mathbf{7 5}$ \\
\multirow{2}{*}{ L50S } & Média das notas & $7,5^{\mathrm{Aa}}$ & $7,4^{\mathrm{Aa}}$ & $7,2^{\mathrm{Aa}}$ & $7,2^{\mathrm{Aa}}$ & $7,7^{\mathrm{Aa}}$ & $\mathbf{7 , 4}$ \\
& IA\% & 83 & 82 & 80 & 81 & 85 & $\mathbf{8 2}$ \\
\multirow{2}{*}{ L50SK } & Média das notas & $7,5^{\mathrm{Aa}}$ & $7,5^{\mathrm{Aa}}$ & $7,4^{\mathrm{Aa}}$ & $7,4^{\mathrm{Aa}}$ & $7,9^{\mathrm{Aa}}$ & $\mathbf{7 , 5}$ \\
& IA\% & 84 & 83 & 82 & 83 & 87 & $\mathbf{8 4}$ \\
\hline
\end{tabular}

IA\% (índice de aceitabilidade).

Letras maiúsculas (colunas) e letras minúsculas (linhas) iguais não diferem entre si pelo teste de Tukey a $5 \%$ de significância.

A redução do sal em produtos cárneos leva à menor extração de proteínas miofibrilares e, consequentemente, ocorre decréscimo na retenção de água e da cor (PEREDA, et al. 2005). Isso pode ser notado pela avaliação da cor das linguiças, pois a média da nota da amostra com menor concentração de sal (L0), tanto para o sabor quanto para a consistência, foi menor que para as outras duas amostras

Embora não exista relação entre a redução de sal e a diminuição do aroma, percebeu-se a tendência dos julgadores em preferir o aroma das amostras L50S e L50SK. Isso provavelmente se deve à relação entre os sentidos de gustação, olfação e visão (GUYTON, 1998).

O tempero e o sabor estão intimamente relacionados. Assim, observou-se ligeira tendência de elevação das notas da amostra L50SK em relação às amostras L0 e L50S. De acordo com Ramundo et al. (2005), esse resultado se deve ao sal somado à maior adição de pimenta na formulação da amostra L50SK (mais próxima da formulação comercial).

$\mathrm{O}$ índice de aceitabilidade (IA) das três amostras de linguiça toscana, que variou de 70 a 87\% (Tabela 1), foi excelente (considera-se repercussão favorável quando o índice de aceitabilidade for $\geq 70 \%$ ). Segundo Correia et al. (2001), a aceitabilidade representa o principal ponto crítico na elaboração de novo produto para o mercado.

Entre as três amostras analisadas verificou-se grande preferência pela L50SK $(44,29 \%)$ e pela L50S (42,86\%) com teores de sal reduzidos em $50 \%$.

A grande preocupação ao se desenvolver novo produto envolve a intenção de compra pelo consumidor (SANTANA et al., 2006; BISPO et al., 2004). Os resultados obtidos no presente estudo revelaram que $79 \%$ dos participantes certamente comprariam o produto e $19 \%$ provavelmente o comprariam caso estivesse à venda. Resultado considerado satisfatório, indicando que a alteração proposta na elaboração da linguiça frescal foi aprovada pelos consumidores potenciais de carne suína.

\section{CONCLUSÃO}

Verificou-se que a região oeste do Paraná pode ser considerada potencial consumidora de carne suína, sendo que o consumo de embutidos é maior que o de carne in natura.

Quanto à característica sensorial das amostras de linguiça toscana com baixo teor de 
sódio, constatou-se índice de aceitabilidade acima de $70 \%$ para todas as formulações, porém a escala ideal de sabor apontou a amostra L50SK como a mais próxima do ideal (preferida entre os julgadores).

Entende-se que a indústria alimentícia deve cada vez mais investir na elaboração de produtos diferenciados, com melhor valor nutricional e que atendam às necessidades de grupos específicos como o de hipertensos.

\section{ABSTRACT \\ TOSCANA SAUSAGE WITH LOW-SODIUM CONTENT SENSORIAL CHARACTERIZATION AND ANALYSIS OF PORK MEAT AND ITS DERIVATIVES CONSUMPTION IN THE WEST REGION OF PARANÁ STATE (BRAZIL)}

The objective of this study was to verify the acceptability of toscana sausage with low- sodium content, as well as to evaluate the consumption of pork meat and its derivatives in the West Region of Paraná State (Brazil). Three toscana sausage samples with different concentrations of sodium were evaluated by seventy non-trained judges, using the following sensorial tests: hedonic scale, preference and acceptability, flavor ideal scale and purchase intention. The consumption of pork meat was assessed by means of questionnaire containing thirteen semi structured questions. All samples presented acceptability higher than $70 \%$ considering the characteristics evaluated. The most acceptable sample was the one with $50 \%$ of light salt (potassium chlorate) addition, witch achieved $79 \%$ of purchase intent. It was also verified that the West Region of Paraná State is a potential consumer of pork meat, presenting higher consumption of its derivatives than pork "in natura".

KEY-WORDS: SAUSAGE; SODIUM; SENSORY ANALYSIS.

\section{REFERÊNCIAS}

1 ABIPECS. Associação Brasileira da Indústria Produtora e Exportadora de Carne Suína. Consumo de carne suína. 2007. Disponível em: <http::// www.abipecs.org.br/>. Acesso em 01 nov. 2007.

2 ALMEIDA, O.C. Avaliação físico-química e microbiológica de lingüiça toscana porcionada e armazenada em diferentes embalagens, sob condições de estocagem similares às praticadas em supermercados. 2005, 80 p. Dissertação (Mestrado em Tecnologia de Alimentos), Faculdade de Engenharia de Alimentos, Universidade Estadual de Campinas (UNICAMP), Campinas, 2005.

3 BERNARDI, D.M.; ROMAN, J.A. Linguiça toscana com redução no teor de sódio: caracterização microbiológica, físicoquímica e nutricional. Revista Nutrição Brasil. No prelo, 2011.

4 BISPO, E.S.; SANTANA, L.R.R.; CARVALHO, R.D.S.; ANDRADE, G.; LEITE, C.C. Aproveitamento industrial de marisco na produção de lingüiça. Revista Ciencia e Tecnologia de Alimentos, v. 24, n. 4, p. 664-668, 2004.

5 BRAGAGNOLO, N.; RODRIGUEZ-AMAYA, D.B. Teores de colesterol, lipídios totais e ácidos graxos em cortes de carne suína. Revista Ciência e Tecnologia de Alimentos, v. 22, n. 1, p.98-104, 2002.

6 BRASIL. Ministerio da Agricultura e Abastecimento. Instrução normativa n.4, de 31 de março de 2000. Regulamentos técnicos de identidade e qualidade de carne mecanicamente separada, de mortadela, de lingüiça e de salsicha. Diário Oficial [da] Republica Federativa do Brasil, Brasilia, 05 abr. 2000, Seção 1, p. 6.

7 CABRAL, P.C.; MELO, A.M.C.A.; AMADO, T.C.F.; SANTOS, R.M.A.B. Avaliação antropométrica e dietética de hipertensos atendidos em ambulatório de um hospital universitário. Revista de Nutrição, v. 16, n.1, p.61-71, 2003.

8 CASTRO, I.; BATLOUNI, M.; CANTARELLI, E.; RAMIRES, J.A.F; LUNA, R.L.; FEITOSA, G.S. Cardiologia: princípios e prática. Porto Alegre: Artes Médicas, 1999.

9 CORREIA, R.T.P.; MENDONÇA, S.C.; LIMA, M.L.; SILVA, P.D. Avaliação química e sensorial de lingüiças de pescado tipo frescal. Boletim do CEPPA, v. 19, n.2, p.183-189, 2001.

10 FARIA, I.G.; FERREIRA, J.M.; GARCIA, S.K. Mercado consumidor de carne suína e derivados em Belo Horizonte. Revista Arquivo Brasileiro de Medicina Veterinária e Zootecnia, v. 58, n.2, p.251-256, 2006.

11 FERRARI, C.C.; SOARES, L.M.V. Concentrações de sódio em bebidas carbonatadas nacionais. Revista Ciência Tecnologia de Alimentos, v. 23, n.3, p.414-417, 2003.

12 GUYTON, A.C. Fisiologia humana. 6. ed. Rio de Janeiro: Guanabara Koogan, 1998. 
13 KATER, C.E.; COSTA-SANTOS, M. O espectro das síndromes de hipertensão esteróide na infância e adolescência. Arquivos Brasileiros de Endocrinologia e Metabolismo, v. 45, n.1, p. 76-86, 2001.

14 MOLINA, M.D.C.B.; CUNHAB, R.S.; HERKENHOFFB, L.F.; MILLB, J.G. Hipertensão arterial e consumo de sal em população urbana. Revista Saúde Pública, v. 37, n.6, p. 743-750, 2003.

15 MONTEIRO, C.L.B. Técnicas de avaliação sensorial. 2.ed. Curitiba: CEPPA-UFPR, 1984.

16 PEREDA, J.A.O.; RODRÍGUES, M.I.C.; ÁLVAREZ, L.F.; SANZ, M.L.G. Tecnologia de alimentos: alimentos de origem animal. Sao Paulo: Artmed, 2005. v.2.

17 RAMUNDO, A.; COUTO, S.M.; LANZILLOTTI, H.S. Elaboração e análise sensorial de lingüiças caseiras. Revista Higiene Alimentar, v. 128, n.19, p.70-77, 2005.

18 REIS, N.T.; COPLE, C.S. Nutrição clínica na hipertensão arterial. Rio de Janeiro: Revinter, 1999.

19 ROPPA, L. Carne suína: mitos e verdades. São Paulo, 2002. Disponível em: <http:://www.porkworld.com.br >. Acesso em: 20 out. 2007

20 SANTANA, L.R.R.; SANTOS, S.C.L; NATALÍCIO, M.A.; MONDRAGON-BERNAL, O.L.; ELIAS, E.M.; SILVA, C.B.; ZEPKA, L.Q.; MARTINS, I.S.L.; VERNAZA, M.G.; CASTILLO-PIZARRO, C.; BOLINI, H.M.A. Perfil sensorial de iogurte light, sabor pêssego. Revista Ciência e Tecnologia de Alimentos, v. 26, n.3, p.619-625, 2006.

21 STATSOFT INC. Statistics for Windows [Computer program manual]. Tulsa, 1995.

\section{Anexo 1 - MODELO DE QUESTIONÁRIO USADO NA PESQUISA}
Idade (anos):
Gênero: ( ) Feminino
( ) Masculino

Descendência: ( ) Italiana ( ) Portuguesa （ )Alemã ( ) Japonesa ( ) Outras

\section{Profissão:}

1) Você consome carne suína?

( ) $\operatorname{sim}$

( ) não

2) Seu consumo de carne suína in natura (fresca) é?

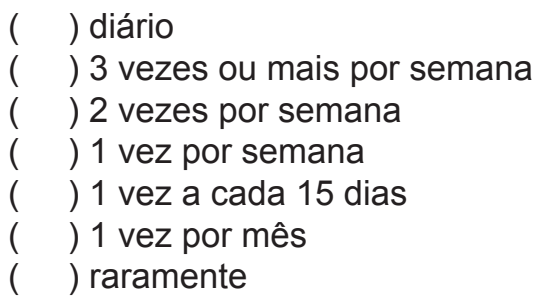

3) Seu consumo de embutidos é?

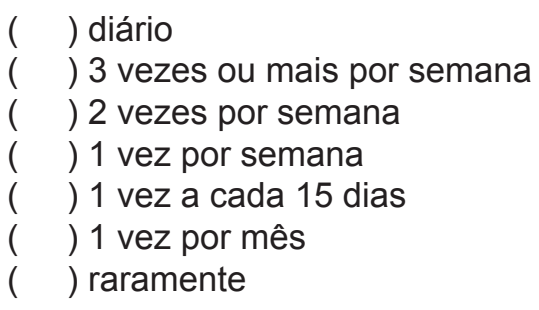


4) Você considera que consome linguiça frescal em que quantidade?

Qual a frequência?

( ) raramente

( ) 1 vez por mês ou menos

( ) 1 vez a cada 15 dias

( ) 1 vez por semana

( ) 3 vezes ou mais por semana

( ) diariamente

5) Indique o principal motivo pelo qual você consome carne suína?
( ) saudável
( ) variar o cardápio
( ) hábito familiar
( ) preço
( ) gosta
( ) praticidade
( )
( ) maciez, sabor

6) Dos produtos de carne suína, qual (is) você consome?
( ) nenhum
( ) costela
( ) salame
( ) bacon
( ) linguiça fresca
( ) defumados em geral
( ) salsicha
( ) pernil
( ) lombo
( ) presunto
( ) cracóvia
( )

7) A carne suína tem grande quantidade de gordura?
( ) $\operatorname{Sim}$
( ) Não
( ) Não quero opinar

8) Você já ouviu falar de alguma contra-indicação para a ingestão de carne suína?

( ) não

( ) sim, qual?

9) Para uma dieta equilibrada, quantas vezes ao dia uma pessoa deve ingerir carnes e derivados?
( ) não sei
( ) nenhuma vez
( ) 01 vez
( ) 02 vezes
( ) 03 vezes
( ) 05 ou mais vezes

10) Alguma vez você precisou deixar de ingerir alimentos com muita quantidade de sal?
( ) $\operatorname{Sim}$
( ) Não

11) Algum médico já diagnosticou que você tem "pressão alta"?
( ) $\mathrm{Sim}$
( ) Não
( ) Não quero opinar 
12) Você atualmente toma remédio para pressão?

( ) Sim
( ) Não
( ) Não quero opinar

13) Em sua família existe algum caso de "pressão alta"?

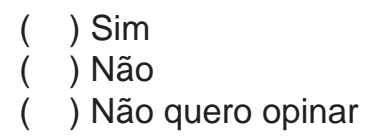

Se a resposta foi sim, qual o grau de parentesco? 